\title{
UNA NUEVA PERSONALIDAD PARA JUAN MELCHOR PÉREZ, SCULTORE E TRAGETTATORE AL SERVICIO DEL VII DUQUE DE MONTALTO Y JUAN JOSÉ DE AUSTRIA*
}

\author{
YOLANDA Gil SAURA ${ }^{1}$ \\ Universitat de València
}

\begin{abstract}
El artículo presenta a Juan Melchor Pérez (doc. 1642-1650) como un escultor y fundidor sucesivamente al servicio del Duque de Montalto y Juan José de Austria, cuestiona la identificación de uno de los bustos de bronce firmados por él que se conserva en el Museo del Prado, se plantea la complejidad de determinar la autoría en las obras realizadas en bronce y el papel de los escultores de corte al servicio de familias nobles.
\end{abstract}

Palabras clave: escultura; fundición; VII Duque de Montalto; Juan José de Austria; retratos; bronce.

\section{A NEW PERSONALITY FOR JUAN MELCHOR PÉREZ, SCULTORE E TRAGETTATORE SERVING THE $7^{\text {TH }}$ DUKE OF MONTALTO AND JUAN JOSÉ DE AUSTRIA}

This article introduces Juan Melchor Pérez (doc. 1642-1650) as a sculptor and foundry artisan successively working for the Duke of Montalto and Juan José de Austria, and questions the identification of one of the bronze busts signed by him in the Prado Museum. The author likewise explains the complexity in determining the authorship of works in bronze, and the role of court sculptors in the service of the nobility.

Key words: sculpture; foundry; $7^{\text {th }}$ Duke of Montalto; Juan José de Austria; portraits; bronze.

Cómo citar este artículo / Citation: Gil Saura, Yolanda (2018): "Una nueva personalidad para Juan Melchor Pérez, scultore e tragettatore al servicio del VII Duque de Montalto y Juan José de Austria". En: Archivo Español de Arte, vol. 91, núm. 362, Madrid, pp. 101-112. https://doi.org/10.3989/aearte 2018.07.

Triste es no poder llegar a una solución definitiva, ya que la severidad en la investigación y el culto de la verdad prohíbe formular juicios con simples indicios que son siempre aventurados, pero bueno es, por lo menos, plantear el problema y declarar que lo que se daba como cosa sabida no lo es².

Estas fueron las palabras de Enrique Serrano Fatigati, director de la Sociedad Española de Excursiones, cuando al redactar en 1909 su trabajo sobre la escultura madrileña tuvo que enfrentarse a los dos bustos de bronce que entonces se creía representaban al Conde Duque de Olivares y Don Juan José de Austria. Hacía poco tiempo que habían sido trasladados al Museo del Prado

* Este trabajo se inserta en el Proyecto Ecos Culturales artísticos y arquitectónicos entre Valencia y el Mediterráneo en Epoca Moderna (HAR2014-54751-P).

1 Yolanda.gil@uv.es / ORCID iD: http://orcid.org/0000-0002-8230-3632.

2 Serrano Fatigati, 1909: 201-233. 
desde la Academia de San Fernando y la catalogación de Eduardo Barrón había sacado a la luz las firmas, IO:MEL:PERES F 1643 IOVANI MELCHIOR PERES FECIT IN NEAP. 1648.

El descubrimiento de las firmas y fechas implicaba desestimar la autoría de Pietro Tacca apuntada por Ceán Bermúdez y obligaba a los especialistas a intentar otorgar identidad a un nuevo personaje, Juan Melchor Pérez o Giovanni Melchiorre Peres, ante el que Serrano manifestaba su perplejidad, o al menos su curiosidad: "Es extraño que no sea muy conocido el autor de unas obras que revelan todavía más maestría que genio, que no han podido ser, por lo tanto, el fruto de la inspiración de un momento, saliendo por ellas Juan Melchor Peres de la obscuridad y volviendo a ella una vez producidas" 3

El descubrimiento de las fechas de ejecución no solamente desestimaba la autoría de Pietro Tacca y ponía sobre el tablero a un artífice totalmente desconocido, sino que llegaba a replantear las identificaciones. Es Elías Tormo el que ese mismo año y en las mismas páginas propone una nueva identificación para el primer retrato. En un primer momento se inclinó por el marqués de Leganés, pero finalmente propuso la identificación con quien ocupaba el virreinato napolitano en 1643, el Duque de Medina de las Torres, Ramiro Núñez de Guzmán ${ }^{4}$.

Resuelto en principio el problema de la identificación, al año siguiente Tormo escribió otro artículo redactando una "Nota sobre Giovanni Melchior Peres". Continuando con la perplejidad que ya había manifestado Serrano, Tormo tuvo que confesar que no había conseguido encontrar su nombre en los principales repertorios italiano y por ello concluyó que debía tratarse de un escultor discípulo de un todavía casi desconocido Finelli5.

De Giuliano Finelli (1602-1653) se sabía entonces que era un escultor de Carrara educado en Nápoles que había tenido a su cargo la realización de las grandes estatuas de bronce para la capilla del tesoro de San Genaro. Para fundir esas estatuas Finelli había hecho venir de Roma a al ya anciano Gregorio de Rossi y al joven sobrino de Finelli, Domenico Guidi. La hipótesis de Tormo era que, Juan Melchor Pérez sería un tercero ${ }^{6}$.

En lo sustancial la historiografía ha venido sosteniendo hasta hoy conclusiones muy similares a las que asentaron Serrano y Tormo, aunque ha planteado muchas menos incógnitas de las que ellos dejaron abiertas. Es Tormo el que sitúa a Pérez en la escuela napolitana, lo vincula al taller de escultura en bronce que estaba trabajando en la capilla de San Genaro y acaba considerando la posibilidad de que se trate de un especialista en fundición. La escasez de datos sobre la figura de Pérez ha hecho que de manera categórica se haya considerado a Juan Melchor Pérez un fundidor de origen español instalado en Nápoles vinculado al círculo de Finelli.

Tras los trabajos de Barrón, Serrano Fatigati y Tormo, han sido citadas por Estella y González Palacios, la atención más detenida la han recibido de Coppel y sobre todo ha sido Dombrowski en su monumental trabajo sobre Giuliano Finelli quien las ha estudiado de manera más detallada ${ }^{7}$. En todos los casos se ha aceptado la identificación propuesta por Tormo para el primer retrato y se ha relegado la figura de Juan Melchor Pérez a la de un fundidor al servicio de Finelli. Partiendo de esas bases, Dombrowski ha intentado delimitar la responsabilidad de Finelli y de Mencaglia en cada una de las obras, atribuyendo a Finelli el supuesto rostro del duque de Medina de las Torres y a su ayudante Mencaglia el busto completo de Don Juan José de Austria.

\footnotetext{
3 Serrano Fatigati, 1909: 201-233.

4 Tormo, 1909: 291-312 (especialmente a partir de la p. 299, "El busto en bronce de un Guzmán en el Museo del Prado")

5 "Si la rebusca no la hubiera hecho a la vez que la de Nacherino, hoy tan conocido, habría que pensar que me equivoqué al ver, con tan sincera evidencia, firmas de escultor y no de fundición, en los dos bustos del Museo del Prado", Tormo, 1910: 113-126.

6 "Verosímilmente Melchior Peres debió ser un tercero entre ellos, ya que por entonces firma (1643) uno de los bustos del Prado, y en Nápoles el otro en 1648, demostrándose de todas suertes la existencia en aquella ciudad y aquellos años de fundiciones escultóricas notables, con numeroso personal de artistas", Tormo, 1910: 113-126.

7 Dombrowski, 1997: 384-387. Coppel Aréizaga, 1998: 140-142 y 491. Dombrowski propone la autoría de Finelli y Mencaglia para el busto de Medina de las Torres, y Mencaglia en solitario para el de don Juan José de Austria.
} 
Este estado de la cuestión solamente se ha modificado muy recientemente. En el Museo del Prado Leticia Azcue - jefa de conservación de escultura y artes decorativas - pudo comprobar que las fechas leídas por Barrón y que se habían sostenido hasta ahora no eran del todo correctas, y que las dos piezas estaban firmadas en la misma fecha, $1648^{8}$.

Con este artículo nos proponemos hacer una relectura de algunos documentos recientemente publicados sobre Juan Melchor Pérez y la nueva datación replanteando algunas de las cuestiones que ya dejaron abiertas Serrano y Tormo como la personalidad de los retratados, la diferenciación del papel de escultores y fundidores en la escultura en bronce o el significado de la firma en este tipo de escultura. Juan Melchor Pérez se nos presentará así como un artífice especializado en el trabajo del bronce sucesivamente al servicio del VII Duque de Montalto y de Don Juan José de Austria.

Algunos indicios nos llevan a proponer para el primero de los retratos —el hasta ahora considerado de Medina de las Torres - como un retrato del VII Duque de Montalto, pero al igual que Serrano no llegaremos a soluciones definitivas. En todo caso y volviendo otra vez a sus palabras: "Lo que sí puede afirmarse es que estos dos bustos, en unión de la estatua ecuestre de Felipe IV, son las tres obras más hermosas en bronce y de procedencia extranjera que vinieron en el siglo XVII a unirse a las producidas en el país y enriquecer los tesoros artísticos madrileños, que no resultan tan pobres cuando se les estudia como parece cuando se les desconoce".

\section{La procedencia}

Los dos bustos que nos ocupan llegaron al Museo del Prado desde la Real Academia de Bellas Artes de San Fernando, y allí se señala su procedencia de la colección real.

La documentación es mucho más clara en el caso del busto de Juan José de Austria. Ahora sabemos que el busto formaba parte de la colección personal de Don Juan José que debió ser quien lo encargó. Cuando fue nombrado Gobernador de los Países Bajos en 1656 Velázquez fue el encargado de revisar parte de su colección y seleccionó el busto como una de las piezas que debía trasladarse al Alcázar. Los inventarios lo localizan en las Bóvedas del Tigre en 1686, descrito como "Otra estatua medio Cuerpo del Sor. Dn. Juan de Austria Armado y una Banda todo de Bronce y su repisa de mármol negro". Del Alcázar pasó al nuevo Palacio Real y de ahí a la Academia de San Fernando.

Los inventarios de la Academia de San Fernando presentan siempre las dos piezas juntas. El inventario de 1817 las recogía en la denominada "Sala del Recibimiento", luego conocida como "Sala segunda" como "Busto de bronce con peana de mármol de Don Juan de Austria, hijo de Don Felipe IV" y "Busto del conde Duque de Olivares, de bronce con peana de mármol"9. Los catálogos de 1818 y 1821 mantienen el lugar y las identificaciones, solamente el catálogo de 1824 -inspirado por Ceán Bermúdez - se atreve a atribuirlas a Pietro Tacca. En 1829 las esculturas pasaron al Museo del Prado y fue allí donde Eduardo Barrón, al hacer el primer catálogo de la escultura del museo en 1908, leyó las firmas en ambas ${ }^{10}$.

\footnotetext{
${ }^{8}$ Leticia Azcue revisó las obras al redactar la ficha del retrato de Juan José de Austria con motivo de la exposición El Arte del Poder de 2010. Azcue, 2010: 214-15. La reelaboración de la ficha descriptiva del otro retrato se hizo seguidamente a nivel interno, y la modificación de la fecha en la web del museo, cuya actualización ha progresado mucho en los últimos tiempos, se llevó a cabo en el año 2016. Agradezco a Miguel Falomir y Letizia Azcue su generosidad, sus observaciones y las facilidades para el estudio de la pieza.

9 Catálogo, 1817: 8-9.

10 IO:MEL:PERES F 1643 y IOVANI MELCHIOR PERES FECIT IN NEAP. 1648. Barrón, 1908: 181 y 204. En el que entonces se suponía del Conde Duque, la inscripción se sitúa bajo la hombrera derecha de la armadura, en el de Don Juan José de Austria aparece en un borde del lazo de la banda. Ahora sabemos que en los dos casos la fecha es 1648 .
} 


\section{Juan Melchor Pérez al servicio del duque de Montalto}

La primeras noticias que conocemos del personaje que nos ocupa datan de 1642, en mayo y julio de ese año sendos listados de criados del VII duque de Montalto publicados por Barbara Mancuso y Giovanni Mendola citan a "Juan Melchor Pérez, escultor"11. El dato se confirma con la noticia recientemente aportada por Giuseppe Giugno que lo ha documentado ese mismo año tasando una "grada d'altare" realizada por Pietro del Monte para el palacio del duque en Caltanissetta (Sicilia), una pieza que años después sería descrita como "una grada di ferro grande con sua gelosia di legname"12.

Luigi Guglielmo de Moncada y Aragón, VII duque de Montalto (1614-1672) — conocido en los últimos años de su vida como el cardenal Moncada-, por parte de padre procedía de la nobleza napolitana y siciliana donde radicaban la mayor parte de sus posesiones, por parte de madre era nieto de los duques de Medinaceli y él mismo se casó con apenas quince años con María Afán de Ribera y Moura, hija del III Duque de Alcalá, por entonces nombrado virrey de Nápoles. Tras una infancia en Palermo, el joven Moncada se instaló en Nápoles con su suegro que se convirtió en su tutor y de él recibió sin duda una exquisita educación artística. Cuando el duque de Alcalá fue nombrado virrey de Sicilia en 1632 la pareja se trasladó con él y entre 1635 y 1639, tras la marcha de su suegro a Flandes, gobernó Sicilia en calidad de presidente del reino. En 1637 murió su suegro y en 1639 su esposa, el duque se instaló temporalmente en Roma en la embajada de España acogido por el tío de su esposa, el II marqués de Castelrodrigo. De Roma marchó a su palacio de Caltanissetta, en Sicilia, allí preparó la partida para España en julio de 1642 para ponerse al servicio del monarca y casarse por segunda vez, esta vez con Catalina Moncada, hija del marqués de Aytona ${ }^{13}$.

Juan Melchor Pérez aparece a sueldo del duque precisamente en esa estancia en Caltanissetta antes del traslado a Madrid. Junto a Pérez aparecen otros artistas, entre ellos algunos pintores que iban a acompañar al duque en sus futuros desplazamientos como virrey a Cerdeña (1644-49) o Valencia (1653-59). Todos debían ser artistas a sueldo, artistas de casa o de corte, que de alguna manera formaban parte de la "familia" y que en algunos casos, como muchos otros criados, eran heredados del Duque de Alcalá. La presencia de este tipo de pintores o escultores, mezclados con músicos, historiógrafos, perfumistas o profesores de danza era habitual en estas cortes itinerantes y su presencia a sueldo no impedía el contrato de otros pintores de renombre como era el caso de Ribera, quien sabemos ya había retratado al duque en sus años napolitanos.

En 1642 Juan Melchor Pérez debía ser un "escultor de casa" del Duque de Montalto, no sabemos nada de su formación ni del momento en que entró a formar parte de ella, ni tampoco cuando la abandonó. El nombre italianizado ha hecho pensar que se trata de un artífice de origen español. Pudo entrar en su casa en los años napolitanos acompañando al duque de Alcalá, en torno a 1630, o en los años del gobierno de Sicilia cuando desplegó un importantísimo programa de obras públicas ${ }^{14}$, o incluso — dado que no se han publicado otras noticias anteriores - se podría haber incorporado durante el período en que Montalto había estado en Roma. En 1642 la documentación hace referencia claramente a Pérez como escultor — por tres veces en tres documentos distintos - no sabemos de ninguna obra realizada por él, pero la obra que debe visurar y tasar en Caltanissetta es una pieza de metal, el material en el que parece ser especialista.

11 Mancuso, 2006: 85-152. Mendola, 2006: 153-175. Mancuso y Mendola publican sendos ensayos en el mismo volumen, hacen referencia a diferentes volúmenes del Archivo Moncada y en los dos casos aparece Pérez como escultor al servicio del duque. Hemos revisado estos listados, Juan Melchor Pérez aparece junto al pintor Henrrique Brant y el platero Juan de Gregorio, cobrando una cantidad considerablemente superior a la que ganaban éstos.

12 Giugno, 2012: 611-612.

13 La figura del VII duque de Montalto ha sido fundamentalmente estudiada por Rafaella Pilo. Véase sobre todo Pilo, 2008 y Scalisi, 2006; 2008a; 2008b.

${ }_{14}$ En esos años están documentados trabajando para el duque los escultores Nunzzio la Mattina, que trabaja en el palacio de la familia en Palermo, el de Aiutamicristo, y Niccola Trabaglia, escultor natural de Carrara padre del más conocido Giovanni Travaglia y abuelo de Giacomo Serpotta, éste se encargó de las esculturas e inscripciones de obras públicas como la Porta Felice. Mendola, 2006. 
No tenemos más noticias de Pérez hasta seis años después. El duque de Montalto se trasladó a la Corte, a Madrid, y no parece que el escultor formase parte de la comitiva. En Madrid volvió a casarse, esta vez con Catalina Moncada y en 1644 el matrimonio se trasladó a Cerdeña donde el duque iba a ocupar el cargo de virrey y vuelve a desarrollar un ambicioso programa artístico. Conocemos un listado de sus criados en 1645 y allí tampoco aparece ${ }^{15}$.

\section{Juan Melchor Pérez, escultor de Don Juan José de Austria}

En 1648 se datan los dos retratos que nos ocupan, el busto de Don Juan José de Austria y el de Medina de las Torres.

El retrato de Don Juan José (fig. 1) se realiza probablemente en uno de los momentos más decisivos de su carrera política. Nombrado en 1647 Generalisimo de los Ejércitos de Mar y poco antes prior de San Juan de Jerusalén, el hijo ilegítimo de Felipe IV llegó a Nápoles el 1 de octubre de 1647 con apenas 18 años, sofocó la revuelta de Massaniello, fue nombrado interinamente virrey en sustitución del duque de Arcos y tras la llegada del nuevo virrey, conde de Oñate, fue recompensado con el virreinato de Sicilia.

En el momento en el que comienza a definirse su personalidad política es importante además la definición de su imagen visual y literaria, una definición en la que debió dejarse poco espacio al azar. Una vez sofocada la revuelta fue necesario crear una narración conveniente de los acontecimientos y adornarla también con testimonios visuales, la narración histórica se encargó a Pier Giovanni Capriata y a su colaborador Rafael de Turri, Ribera realizó su famoso retrato a caballo con la ciudad de Nápoles al fondo conservado en el Palacio Real de Madrid y fue entonces cuando se realizó este busto. Antes de salir de la península, el 4 de junio de 1647, se le había entregado una armadura que su padre había recibido de Isabel Clara Eugenia en 1626, esta armadura debía ser depositaria de un simbolismo impor-

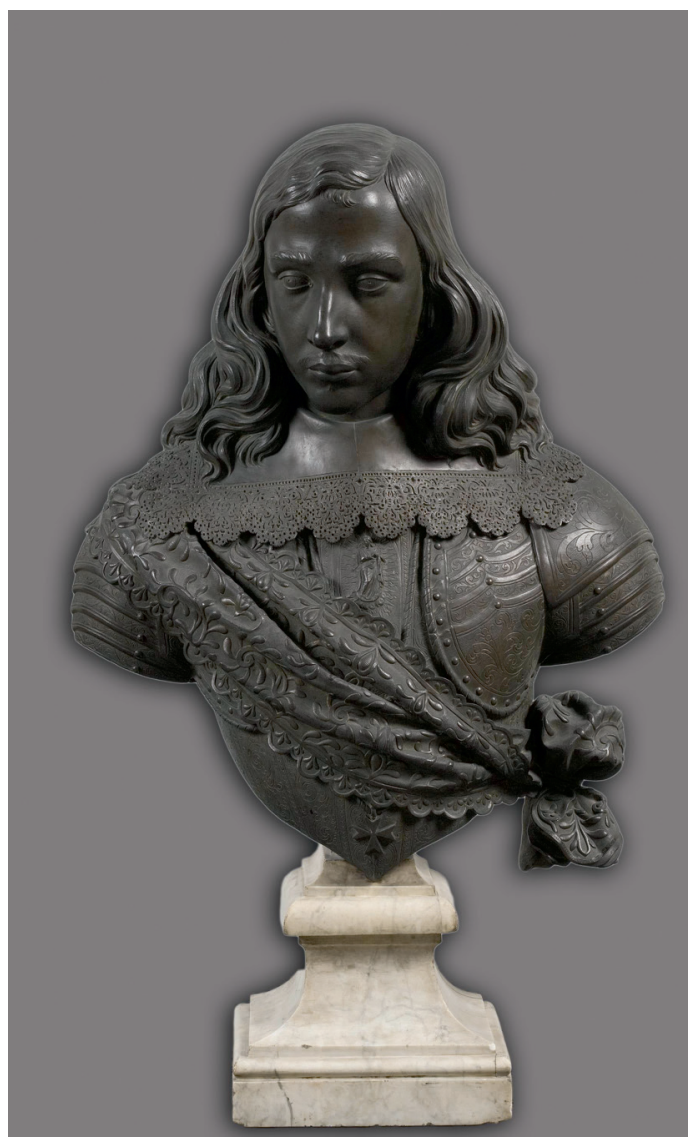

Fig. 1. Juan José de Austria. 1648. Bronce, $80 \times 51 \mathrm{~cm}$. Firmado IOVANI.MELCHOR.PERES./ FECIT.IN.NEAP.1648. Museo del Prado tante y con ella se le retrata ${ }^{16}$.

Ser objeto de una escultura era un privilegio reservado a unos pocos, aunque parece que él estaba casi predestinado, las narraciones de la época relatan que en su trayecto hacia Nápoles apresó la galera "San Diego" que portaba un cargamento de mercancías procedentes de Florencia con destino al rey de Portugal, Juan IV. Entre esas mercancías se encontraban cuatro estatuas que don Juan José hizo enviar a su padre, por entonces se llegó a escribir en la corte que "quien tan presto ganaba estatuas, había nacido para merecerlas"17. En ese sentido Don Juan José debía

15 El listado y las iniciativas del duque en esos años en Manfrè/Mauro, 2016: 183-214.

16 La idenficación de la armadura fue realizada por el Conde de Vindicado, 1898: 112-113.

17 Gonzalez Asenjo, 2006: 48. 
competir con el primer Don Juan, el hijo ilegítimo de Carlos I al que se había levantado una estatua de bronce en Mesina ${ }^{18}$.

El encargo pudo partir del propio Don Juan José o ser un regalo para él, pues la escultura quedó en su poder hasta que - como ya hemos apuntado - en 1652 Velázquez la seleccionó como una de las piezas que debía trasladarse al Alcázar. El busto ostenta la firma, IOVANI MELCHIOR PERES FECIT IN NEAP. 1648, representa a Don Juan José como un joven de 19 años, pelo suelto y bigote, mirada baja, portando la armadura regalada por su padre, valona de encaje, medallón con representación de la Inmaculada y banda de la que pende la Cruz de Malta. El escultor ha prestado sin duda especial atención a los detalles del encaje, el repujado de la armadura y el bordado de la faja.

Dombrowsky publica algunos documentos que creemos no han sido resaltados suficientemente. Después de la realización del retrato de Don Juan José, Pérez debió instalarse en Nápoles de manera más o menos permanente. En 1650, con don Juan José intentando resolver los disturbios en Sicilia, Juan Melchor Pérez aparece en la documentación relativa a las estatuas que Finelli estaba realizando para la Capilla de San Genaro. Pérez es reclamado como experto por Finelli para valorar las estatuas y éste se refiere a él como "Magnifico Giovanni Melchior Perez scultore e tragettatore di S. Altezza serenissima"19. Dejando de lado el significativo tratamiento de "magnífico", es reveladora la clasificación profesional, "scultore e tragettatore" y su vinculación a Su Alteza Serenísima, sin duda don Juan José de Austria ${ }^{20}$.

El hecho de que dos años después de la realización de estos bustos se aluda de esta manera a Pérez puede indicar que éste llegase a tener una vinculación con Don Juan José más permanente. Como sabemos éste tenía unos gustos exquisitos, y sería conocido por su afición a la pintura y su habilidad para la talla, según Juanini: "en la pintura aplicaba con igual maestría el pincel a un perfecto dibujo" y "trabajaba de cincel en oro y planta, como el más experimentado y diestro Maestro"21.

\section{Un retrato sin identificar}

Juan Melchor Pérez es considerado escultor de don Juan José de Austria como antes lo había sido del duque de Montalto, una vez más la documentación solamente lo refleja como experto, pero las obras son de primera magnitud y el tratamiento que le dispensa el escultor más importante de ese momento en Nápoles, Giuliano Finelli, no parece el reservado a un subordinado. Documentado en 1642 en Caltanissetta al servicio del duque de Montalto, en el Prado se conserva otro retrato de busto en bronce, que ahora sabemos datado también en 1648 sin especificar el lugar de fundición (fig. 2).

Como ya hemos recordado la identificación del busto del Museo del Prado como el duque de Medina de las Torres no era más que una hipótesis. Los inventarios de la Real Academia de San Fernando y los primeros del Museo del Prado lo consideraron un retrato del Conde Duque de Olivares, fue Tormo quien lo identificó como Medina de las Torres por ser éste virrey de

18 Las esculturas también podían tener connotaciones negativas, cuando llegó a Nápoles, sus enemigos, "non contenti delle parole, facevano molti ritratti e diverse statue tanto del ré, comoe ancora di D. Gio., e gettandoli nella faccia mille sporchezze, le gettavano poi con inguria nel mare", González Asenjo, 2006: 52.

19 Dombrowski, 1997: 492.

${ }^{20}$ Un año después Finelli alude a Pérez como su fiador. Finelli había tenido que marchar a Roma por orden del conde de Oñate para supervisar los vaciados encargados por Velázquez para el rey, su incumplimiento del contrato en Nápoles hizo que Pérez llegase a ser encarcelado, por ello el 3 de junio de 1651 Finelli desde Nápoles reclamaba al virrey que no podía partir hacia Roma si antes no se le liberaba. Minguito Palomares, 1999: 295-316.

21 Juanini, 1685: 5-6. Agradezco a Joaquín Bérchez las aportaciones sobre la figura de Don Juan José, que en gran medida no tienen cabida en este artículo. 
Nápoles en 1643 en ese año y por la similitud con un busto que poseía el conde de Casasola hoy per$\operatorname{dido}^{22}$.

Siempre hemos considerado que esa era una hipótesis endeble, pero con la nueva lectura de la firma realizada por la conservadora del Museo del Prado, Leticia Azcue, IO:MEL:PERES F 1648, la relación con Medina de las Torres que hacía cuatro años que había dejado de ser virrey de Nápoles deja de tener sentido $^{23}$ (fig. 3).

Mientras creíamos que el busto estaba datado en 1643 contemplamos la posibilidad — con dudas - de que se tratase de un retrato del Duque de Montalto. En un inventario realizado en Caltanissetta en 1652 a la vuelta del virreinato de Cerdeña y antes de trasladarse a Valencia como virrey, se cita "Una statua di brunzo del duca mio signore a mezzo corpo" 24 , que podría identificarse con el busto conservado hoy en el Prado. El gusto del duque por la escultura en bronce no terminaría ahí, investigaciones en curso nos lo presentan años después como un ávido coleccionista que encarga multitud de retratos propios y de sus antepasados, entre ellos una galería de retratos realizados en bronce claramente identificables en los inventarios realizados tras su muerte en Madrid.

Hemos dicho que la identificación de Tormo pretendió justificarse con el parecido a un retrato perdido del duque de Medina de las Torres, cuyo rostro conocemos también por el grabado de muy mala calidad reproducido en la obra de Parrino. Tormo

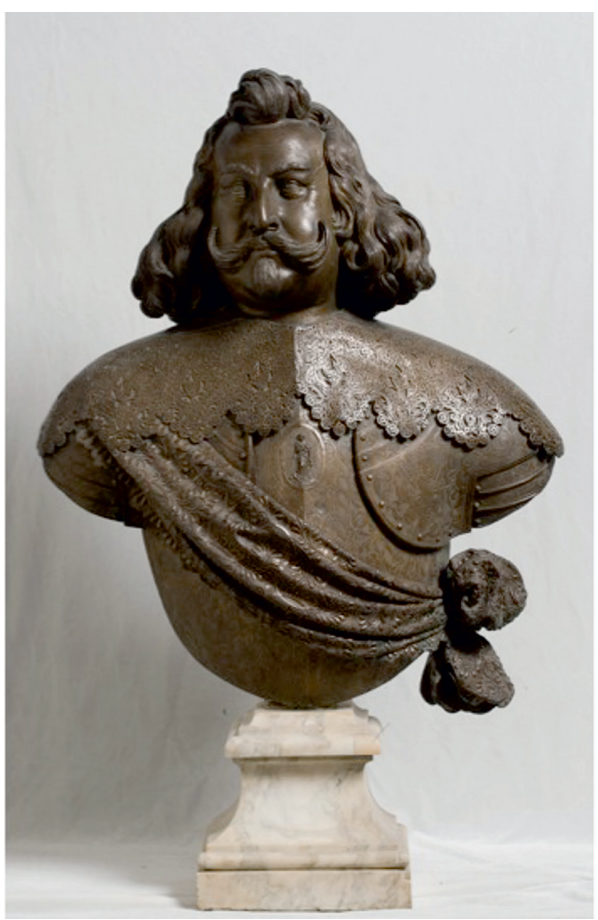

Fig. 2. Retrato de personaje (tradicionalmente identificado como el II Duque de Medina de las Torres). 1648. Bronce, $75 \times 64 \mathrm{~cm}$. Firmado IO:MEL:PERES F 1648. Museo del Prado llega a argumentar el innegable aire de familia de los Guzmanes. El parecido podría existir, pero es bien cuestionable. Milena Viceconte ha publicado una medalla que representa al virrey con mayor verosimilitud, y el parecido no parece tal (fig. 4). Existen otros documentos iconográficos que con el mismo razonamiento, abundarían en la identificación con el duque de Montalto. Se trata de una medalla acuñada en Palermo poco antes de la realización de este retrato ${ }^{25}$ (fig. 5) y de un retrato pintado conservado en una colección privada palermitana que se atribuyó a Pietro Novellii ${ }^{26}$ (fig. 6). Al margen de que en otro lugar rechazamos la atribución y enmarcamos el retrato unos años más tarde en el virreinato valenciano del duque (1653-59), las facciones tanto del retrato como de la medalla pueden ciertamente ser similares a las del busto en bronce conservado en el Prado.

22 Tormo, 1909: 291-312. Se trataba de un busto del segundo Duque de Medina de las Torres presentado en la Exposición de Retratos de 1902. En su contestación Serrano Fatigati llega a plantear que la firma de Juan Melchor Pérez es una falsificación

${ }^{23}$ Recientemente han publicado excelentes estudios sobre Medina de las Torres tanto Fernando Bouza como Milena Viceconte, que le ha dedicado su tesis doctoral, ambos reproducen el retrato conservado en el Prado pero en ningún caso han encontrado rastro de un retrato de este tipo en la documentación. Bouza, 2009: 44-71. Viceconte, 2013.

${ }^{24}$ A.S.Cl, Not. A. Lamammana, vol 650 c. 740, quaderno inserito e cucito nel volume di minute cartulato da c 740 a c. 7535 maggio, 5 ind., 1652, p. 748 r. La transcripción que poseemos de este inventario inédito es fruto de la gentileza de la profesora Zaffuto Rovello. Quisiéramos agradecerle tanto a ella como al arquitecto Vito Migliore, que fue quien nos proporcionó la documentación, esta noticia.

25 Esta medalla fue publicada por Pardo Canalis, 1977: 234-235. En el Museo del Prado se conserva otra versión de la medalla, n. ${ }^{\circ}$ inv. O 1074, agradezco la información a Leticia Azcue.

26 Di Stefano, 1989: 220-221. Reproducido también en Giugno, 2013: 24-32. 


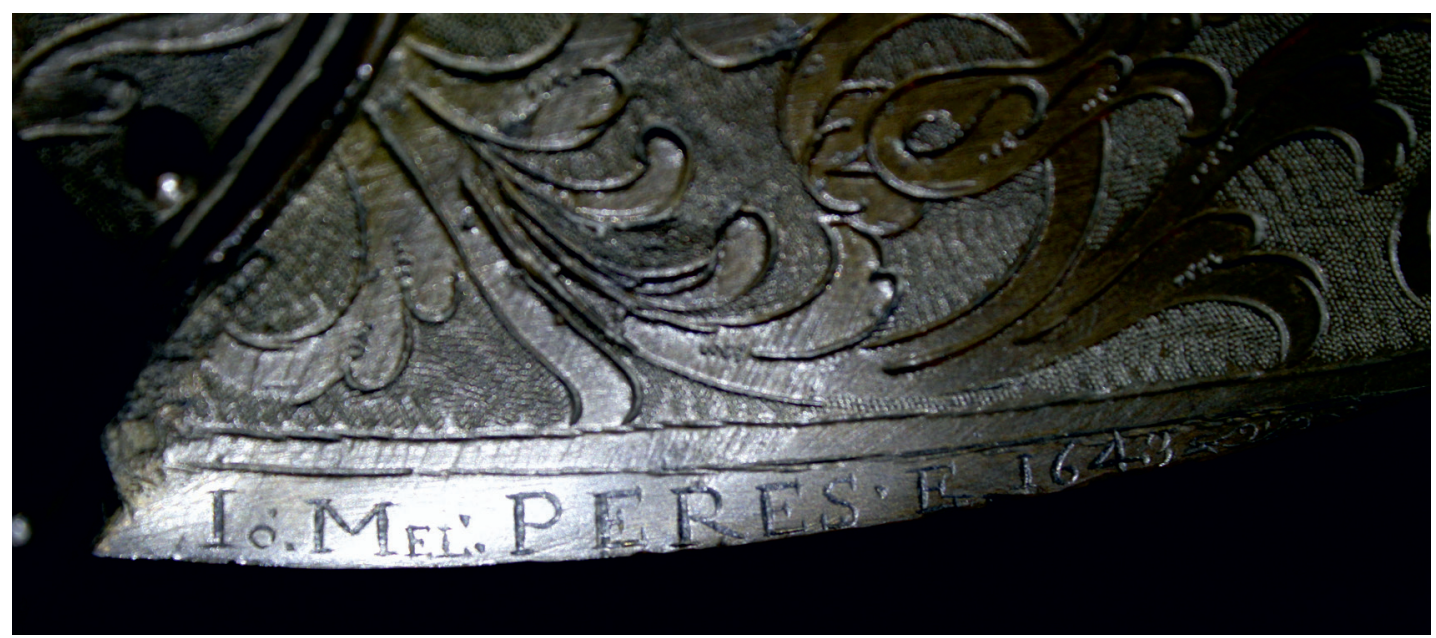

Fig. 3. Detalle de la firma de la fig. 2. Museo del Prado.

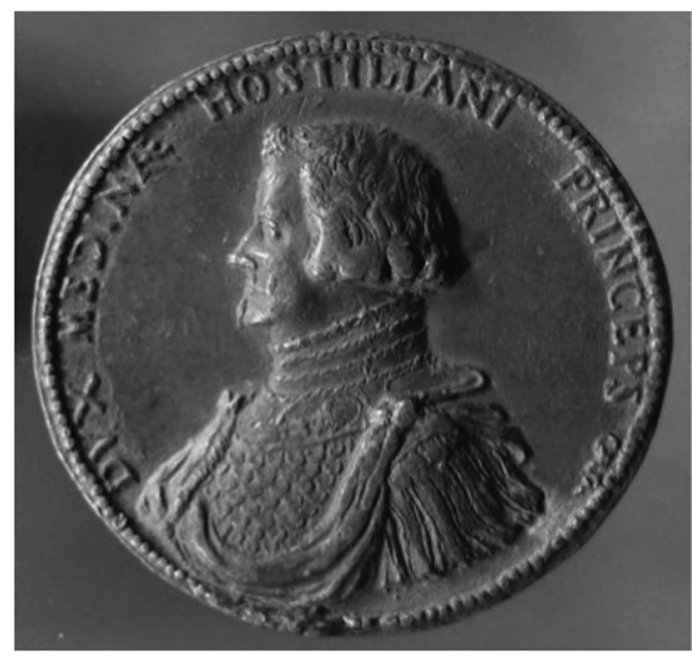

Fig. 4. Medalla conmemorativa de Ramiro Felipe Núñez de Guzmán, II Duque de Medina de las Torres. Inscripción: DVX MEDINAE HOSTILIANI PRINCEPS/1642 NEAPOLI PROREX - SABIONETAE DVX. Museo Arqueológico Nacional.

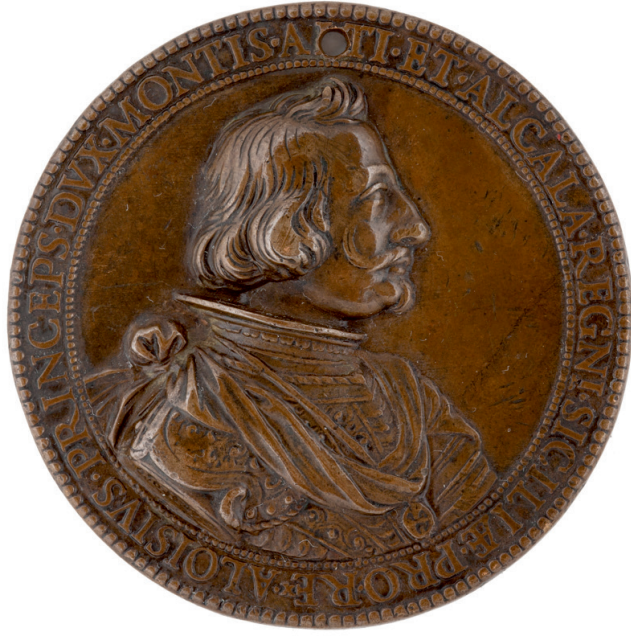

Fig. 5. Medalla commemorativa de Luis Guillermo Moncada, VII Duque de Montalto y Alcalá. Bronce, $59 \mathrm{~mm}$. Inscripción: ALOISIVS. PRINCEPS. DVX. MONTIS. ALTI. ET. ALCALA. REGNI. SICILIAE. PRO. REX/ IN.OMNIBUS.EGO. Museo Lázaro Galdiano

El cambio de fechas provoca otra reflexión, los retratos debieron sin duda partir de una misma iniciativa, encargándose al mismo tiempo. Es difícil dilucidar con quien se quiso equiparar a don Juan José, tal vez con el conde de Oñate, presentándolos a ambos como pacificadores del reino pero las tensiones que vivieron hacen difícil suponer la iniciativa de uno de ellos y el retrato tampoco parece corresponderse con la imagen conocida de Oñate ${ }^{27}$.

Existe una última posibilidad que nos volvería a llevar a la hipótesis inicial, al ser apartado de Nápoles, don Juan José asumió el virreinato de Sicilia, mientras que el duque de Montalto seguía en Cerdeña. Podríamos encontrarnos con un regalo de Montalto que equipara a los dos

27 Minguito Palomares, 2015: 89-123. 


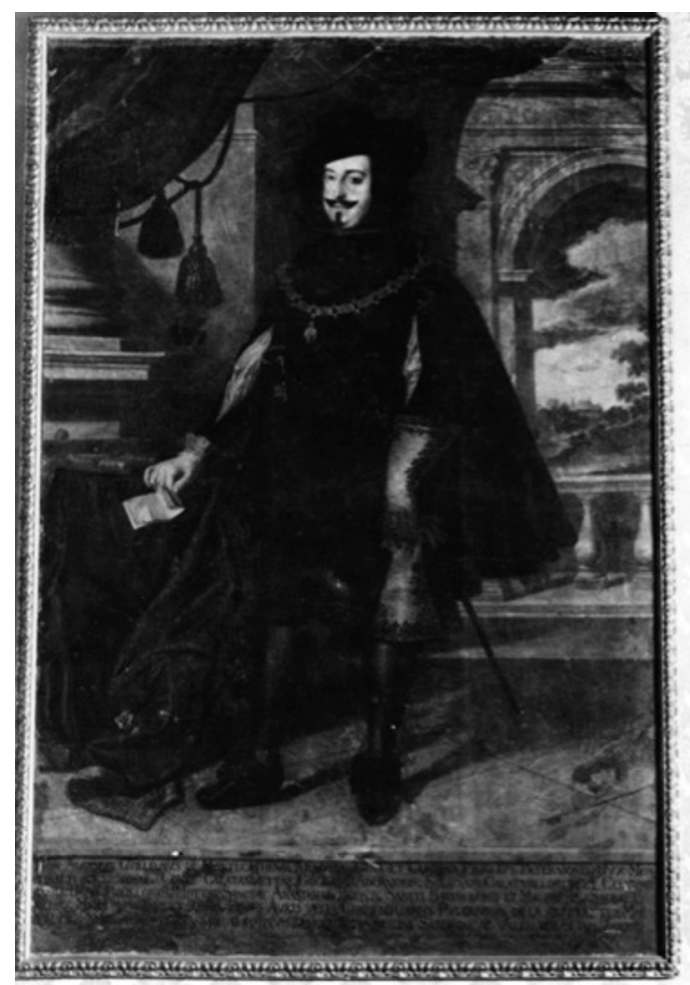

Fig. 6. Retrato del VII Duque de Montalto. Óleo sobre lienzo. Col. privada. Ca. 1653-59.

personajes. Aunque Pérez parece que no estaba ya entre sus criados en Cerdeña podían seguir manteniendo relaciones. Por ahora no nos atrevemos a ir más allá.

Las objeciones existen. Nacido en 1614, en el momento de la realización del retrato el duque debía tener 34 años, el rostro representado en principio nos parece claramente mayor. Como hemos apuntado al principio creemos que la cuestión debe dejarse parcialmente abierta, y que el resto de conclusiones del artículo no quedan invalidadas por esta incógnita.

\section{Scultore e tragettatore}

La de Juan Melchor Pérez sigue siendo una figura llena de lagunas que nos plantea importantes problemas historiográficos. Por un lado el estudio de la abundante nómina de artistas relativamente menores al servicio de familias nobles que figuraban en su casa a manera de criados y cuál fue su verdadero papel en el desarrollo artístico. Por otro, el complejo entramado de artistas involucrados en la realización de la escultura en bronce, la dificultad para deslindar sus tareas y sobre todo el prejuicio a la hora de adjudicar la paternidad de una obra a aquel al que suponemos fue el ejecutor material, una materialidad que parece acercar el trabajo más al de un experto artesano que a lo que algunos consideran un verdadero artista.

La biografía de Pérez sigue siendo una incógnita, su origen y su formación. Una hipótesis verosímil lo podría relacionar con el desconocido escultor español Gaspar Pérez que muere en Roma en $1618^{28}$. El amplio margen temporal hace difícil pensar incluso en una relación de padre e hijo pero tal vez se trate de un taller menor de escultores instalados en Roma vinculados a la clientela española.

En los listados de 1642 Pérez aparece como escultor junto a otros artistas que formaban parte de la casa del duque como "criados". Empezamos a conocer cada vez mejor la amplia nómina de artistas que trabajaron para el duque y sobre todo la relación de dependencia que se estableció con muchos de ellos que pasaron a formar parte de su casa o familia. En el mismo listado en el que aparece Juan Melchor Pérez nos encontramos a Henrico Brant, éste vuelve a aparecer después junto al duque ya convertido en virrey de Cerdeña. Pero más significativa todavía es la noticia que sitúa trabajando en ese palacio de Cagliari realizando los retratos de los virreyes de Cerdeña a "un criado del dicho virrey que se dize don Blas Orliend valenciano" que hemos identificado como el enigmático pintor "Don Blas" vinculado a su suegro, el duque de Alcalá29. En todos los casos se evidenciaría esa continuidad en el recurso a estos artistas vinculados a casas nobiliarias.

La historiografía ha intentado adjudicar a Juan Melchor Pérez otras piezas anónimas, Massimo Pisani vinculó a un posible taller de Pérez un busto de bronce de Gregorio Carafa di Rocce-

\footnotetext{
28 Pomponi, 2011: 107-188.

29 Gil Saura, 2016: 215-224.
} 
lla fechado en torno a $1656^{30}$ y Dombrowski lo relaciona con un busto de bronce conservado en el Museo Filangieri de Nápoles ${ }^{31}$. Las piezas son bien diferentes entre ellas y fundamentalmente tienen en común el tratarse de retratos realizados en bronce supuestamente en el ámbito napolitano.

Es evidente que Pérez es un escultor especializado en el trabajo del metal, de metal era la "grada di ferro" que debe visurar en el palacio de Caltanisetta, de bronce eran las esculturas de la capilla de San Gennaro que también visura como experto y de bronce son las dos esculturas que nos ocupan. Los listados de cuentas de la familia Montalto lo denominan escultor, Finelli hace referencia a él como scultore y tragettatore.

El complejo funcionamiento de los talleres de escultura en bronce, con todas sus incógnitas, ha sido cuidadosamente estudiado por Jennifer Montagu, dejando claro que en este tipo de escultura siempre existen al menos dos artífices, pero pueden ser más, de hecho uno puede dar el diseño, otro realizar el modelo, otro el molde de yeso y otro el fundido en bronce ${ }^{32}$. Las alusiones a Pérez como mero fundidor han parecido rebajar su categoría pero Montagu señala que en ocasiones el fundidor es mencionado como aquel que realiza la escultura aunque no haya realizado el modelo, ya que la realización material se percibía como la etapa más importante y el coste de la fundición era mucho más caro que el modelado ${ }^{33}$. En ese sentido, mientras Cellini otorgaba un carácter casi heroico a la fundición, Adriaen de Vries, otro escultor de corte, delegaba la fundición y sin embargo firmaba las piezas ${ }^{34}$.

Lamouche señala que un mismo fundidor, dependiendo de la ocasión, podía fundir modelos propios o ajenos, y cita como ejemplo a Lodovico del Duca, que se presentaba a sí mismo, como "scultore e tragettatore", también como "tragettatore de metalli" se definía Gregorio de Rossi, que ya anciano fue llamado por Finelli para ocuparse de fundir las esculturas de la capilla de San Genaro que tasaría Pérez. La palabra tragettatore hace referencia al trabajo de un fundidor, pero no es sinónima de fonditore. Lamouche ${ }^{35}$ ha estudiado la utilización de la palabra casi de manera exclusiva en los talleres romanos para referirse a un grupo reducido de fundidores caracterizados por su excelencia técnica y ha advertido sobre la utilización del término escultor aplicado a los fundidores en bronce ${ }^{36}$.

Hasta ahora las aproximaciones a Pérez lo han considerado un fundidor al servicio de Finelli, pero en realidad Pérez aparece tasando la fundición de las estatuas realizada por el veterano De Rossi. Podríamos pensar — como Tormo- que Pérez se instaló en Nápoles precisamente al calor de las obras de la capilla de San Genaro donde un artífice experto en el trabajo del bronce tuvo en esos años un trabajo casi asegurado, pero eso parece casar mal con su papel como experto que parece situarlo al menos en ese momento fuera de la obra.

Tormo pareció no tener dudas de que las firmas que ostentan ambos bustos eran las de un escultor y no las de un fundidor, pero ¿cuál es la diferencia? Cuando se trata de esculturas de plata, el fundidor debe formar parte del gremio de plateros y está obligado a marcar la pieza, esto no sucede así con el bronce. En este caso ¿quién era Juan Melchor Pérez? No estamos en disposición de resolver la incógnita, pero creemos que además de discutir si la firma se corresponde

30 Pisani, 1988: 186-187.

31 Dombrowski, 1991: 386. El busto fue identificado en primer lugar como Francisco Moncada, Dombrowski dudó de la identificación y propuso la de Giovan Battista Ravaschieri. Los inventarios del museo posteriormente publicados por la profesora Nadia Barrella lo señalan como un retrato de la familia Moncada. Barrella, 2010. Aunque en principio nosotros no contemplamos para este busto la autoría de Pérez sí creemos que está vinculado al Duque de Montalto y nos ocupamos de él en otro lugar. Agradecemos a la profesora Nadia Barrella, de la Seconda Università di Napoli su generosa acogida en Nápoles y haber resuelto nuestras dudas sobre la procedencia de la pieza.

32 Montagu, 1999: 4-5.

33 Montagu, 1993: 60.

34 Fogelman, 2008.

35 Quisiera agradecer al profesor Emmanuel Lamouche de la Université de Nantes, su gentileza al permitirme consultar el capítulo de su tesis doctoral, todavía inédita que analiza la utilización en Roma de los términos fonditore y tragettatore y el fluido intercambio de impresiones acerca del tema, Lamouche, 2013.

${ }^{36}$ Lamouche, 2013b: 83-91. 
con lo que nosotros denominamos un escultor o un fundidor, lo que parecería evidente es que la firma es la de aquel que quiso dejar constancia de su responsabilidad sobre la obra y aquel a quien los que la encargaban y costeaban consideraban responsable. No estamos en disposición de saber quienes fueron lo otros responsables de la ejecución, que sin duda existieron, pero no fue a ellos a quien se permitió firmar la obra.

El tema de la firma en los escultores del renacimiento y el barroco ha preocupado sobremanera a los historiadores en los últimos tiempos ${ }^{37}$. Ostrow dedicó un artículo a la aparición de firmas en las esculturas del barroco romano $y$, aunque se limite a considerar ejemplos realizados en mármol, algunas de sus conclusiones no dejan de ser interesantes. Escultores como Bernini, Algardi o Domenico Guidi nunca firmaron una obra, tampoco lo hizo Finelli y sin embargo un desconocido como Pérez al que se ha supuesto subordinado a este último lo hace en dos casos diferentes. Serrano Fatigati ya señaló además que las firmas no son iguales, ello era curioso en unas obras separadas por cinco años como se pensaba hasta ahora, pero lo es más si las obras fueron realizadas en el mismo año, no sabemos incluso si encargadas por el mismo cliente.

Tal vez la firma era una reivindicación de su trabajo como tragettatore, el delicado proceso de fundición de la pieza frente a aquellos que le había proporcionado los modelos o tal vez la reivindicación de una autoría también en la ideación de las piezas. Para Ostrow, la firma es "first and foremost, a declaration of autorship, a signal to both contemporary and future viewers tan the work is his (or her) original creation". En todo caso, es significativo que en unas piezas como los retratos en bronce donde la perpetuación de la memoria del retratado es prioritaria, alberguemos dudas sobre la personalidad de uno de los retratados y sin embargo el artífice ha dejado impresa su firma.

\section{BIBLIOGRAFÍA}

Azcue, Leticia (2010): “Giulio Mencaglia; Juan Melchor Pérez; Juan José de Austria”. En: El arte del Poder. La Real Armería y el retrato de Corte. Madrid: Museo del Prado, pp. 214-215.

Barrella, Nadia (2010): La forma delle idee. Fermenti europei e memoria familiare nel Museo Filangieri di Napoli. Nápoles: Luciano Editore.

Barrón, Eduardo (1908): Museo Nacional de Pintura y Escultura. Catálogo de la escultura. Madrid.

Bouza, Fernando (2009): "De Rafael a Ribera y de Nápoles a Madrid. Nuevos inventarios de la colección Medina de las Torres-Stigliano (1641-1656)". En: Boletín del Museo del Prado, 45, Madrid, pp. 44-71.

Catálogo (1817): Catálogo de los cuadros, estatuas bustos que existen en la Real Academia de San Fernando en este año de 1817. Madrid: Fuentenebro.

Conde de Vindicado (1898): Catálogo histórico-descriptivo de la Real Armería de Madrid. Madrid: Fototipias de Hauser y Menet.

Coppel Aréizaga, Rosario (1998): Catálogo de la escultura de época moderna. Museo del Prado. Siglos XVI-XVIII. Madrid y Santander: Museo del Prado y Fundación Marcelino Botín.

Cupperi, Walter (2012): “'Leo faciebat', 'Leo et Pompeius fecerunt': autorialità multipla e transculturalità nei ritratti leoniani del Prado". En: Schröder, S. (ed.): Leone \& Pompeo Leoni. Madrid: Museo del Prado, pp. 666-84.

Di Stefano, Guido (1989): Pietro Novelli il monrealese. Palermo: Flaccovio.

Dombrowski, Damian (1997): Giuliano Finelli. Bildhauer zwischen Neapel und Rom. Frankfurt am Main: Peter Lang.

Fogelman, Peggy (2008): “The sculptural Personality of Adriaen de Vries". En: Basset, Jane (ed.), The craftsman revealed: Adriaen de Vries, Sculptor in Bronze. Los Ángeles: The Getty Conservation Institute, pp. XI-XIV.

Gil Saura, Yolanda (2016): "Sobre el enigmático pintor 'don Blas' al servicio de los duques de Alcalá y Montalto". En: Pasolini, Alessandra/Pilo, Rafaella (eds.): Cagliari and Valencia during the Baroque Age. Essays on Art, History and Literature. Valencia: Albatros, pp. 215-224.

Giugno, Giuseppe (2012): "Scultura lignea a Caltanissetta nel Seicento tra sacro e profano". En: Pugliatti, T./Rizzo, P./ Russo, P. (eds.): Manufacere et scolpire in lignamine. Scultura e intaglio in legno in Sicilia tra Rinascimento e Barocco. Catania: Giuseppe Maimone Editore, pp. 611-612.

37 El ejemplo más conocido es el de la firma de Miguel Ángel en la Pietà de la basílica de San Pedro, donde éste utilizaría la expresión faciebat tal y como recomendaba Plinio el Viejo y habían hecho Apeles y Polícleto. Sobre esto, con bibliografía anterior, Lavin, 2013: 277-328. En un contexto más cercano, aplicado precisamente a la escultura en bronce, Cupperi, 2012: 666-684. 
Giugno, Giuseppe (2013): "Luigi Guglielmo Moncada: mecenate e uomo político del Seicento". En: Tecla. Rivista di temi di Critica e Letteratura artística, 7, Palermo, pp. 24-32.

González Asenjo, Elvira (2006): Don Juan José de Austria y las artes. Madrid: Fundación de Apoyo a la Historia del Arte Hispánico.

Helmstutler di Dio, Kelley/Coppel, Rosario (2013): Sculpture Collections in Early Modern Spain. Farnham: Ashgate, p. 13.

Herremans, Valerie (2009): Heads on shoulders: Portrait Busts in the Low Countries 1600-1800. Gent: Snoeck.

Lamouche, Emmanuel (2013): Fondeurs, artistas et artisans du bronze à Rome (1585-1625) (Tésis doctoral bajo la dirección de Philippe Sénéchal), Université de Picardie-Jules Verne.

Lavin, Irving (2013): "Divine Grace and the Remedy of the Imperfect. Michelangelo's Signature on the St Peter's Pietà". En: Artibus et historiae. An art anthology, 68, Cracow, pp. 277-328

Mancuso, Barbara (2006): "L'arte signorile d'adeprare le ricchezze. I Moncada mecenati e collezionisti tra Caltanissetta e Palermo (1553-1672)". En: Scalini, Lina (ed.): La Sicilia dei Moncada. Le corti, l'arte e la cultura nei secoli XVIXVII. Catania: Domenico Sanfilippo Editore, pp. 85-152.

Manfré, Valeria/Mauro, Ida (2016): “'Las obras superfluas' di Luigi Guglielmo Moncada. La rappresentazione del potere vicereale a Cagliari nella 'crisi' degli anni Quaranta del Seicdnto". En: Pasolini, Alessandra/Pilo, Rafaella (eds.): Cagliari and Valencia during the Baroque Age. Essays on Art, History and Literature. Valencia: Albatros, pp. 183214

Mendola, Giovanni (2006): "Quadri, palazzi e devoti monasteri. Arte e artista alla corte dei Moncada fra Cinque e Seicento". En: Scalisi, Lina: La Sicilia dei Moncada. Le corti, l'arte e la cultura nei secoli XVI-XVII. Catania: Domenico Sanfilippo Editore, pp. 153-175.

Minguito Palomares, Ana (1999): "El segundo viaje a Italia de Velázquez. Documentos inéditos en el Archivo de Estado de Nápoles". En: Madrid. Revista de arte, geografia e historia, 2, Madrid, pp. 295-316

Minguito Palomares, Ana (2015): "La entrada triunfal del VIII Conde de Oñate en Nápoles: recreación alegórica de un retrato perdido pintado por Jusepe de Ribera". En: Cuadernos de Historia Moderna, 40, Madrid, pp. 89-123.

Montagu, Jennifer (1996): Gold, Silver and Bronze. Metal sculpture of the Roman Baroque. New Haven/London: Yale University Press, pp. 4-5.

Montagu, Jennifer (1993): Roman Baroque Sculpture: The industry of art. New Haven/London: Yale University Press, p. 60 .

Pardo Canalis, Enrique (1977): "Don Luis Guillen de Moncada, duque de Montalto". En: Goya, Madrid, pp. 234-235.

Pilo, Rafaella (2008): Luigi Guglielmo Moncada e il governo della Sicilia (1635-1639). Gli esordi della carriera di un ministro de la Monarquía Católica. Caltanissetta/Roma: Salvatore Sciascia Editore.

Pisani, Massimo (1988): "L’inventario del 1801 per il Palazzo Carafa di Roccella”. En: Napoli Nobilissima. Nápoles, pp. $186-187$

Pomponi, Massimo (2011): "Gli artista presenti a Roma durante il primo trentennio del Seicento nei documenti dell'Archivio Storico del Vicariato". En: Vodret, Rossella (ed.): Alla ricerca di "Ghiongrat" Studi sui libri parrocchiali romani (1600-1630). Roma: "Lerma" di Bretschneider, pp. 107-188.

Scalisi, Lina (2006a): La Sicilia dei Moncada. Le corti, l'arte e la cultura nei secoli XVI-XVII. Catania: Domenico Sanfilippo Editore.

Scalisi, Lina (2006b): “In ómnibus ego. Luigi Guglielmo Moncada (1614-1672”. En: Rivista Storica Italiana, Anno CXX, Fas., II, pp. 503-568.

Scalisi, Lina (2008): La Sicilia degli heroi. Storie di arte e di potere tra Sicilia e Spagna. Catania: Domenico Sanfilippo Editore.

Serrano Fatigati, Enrique (1909): "Escultura en Madrid desde mediados del siglo XVI hasta nuestros días". En: Boletín de la Sociedad Española de Excursiones, Vol. XXVII, 3, Madrid, pp. 201-233.

Simal López, Mercedes (2011): "Nuevas noticias sobre las pinturas para el Real Palacio del Buen Retiro realizadas en Italia (1633-1642)". En: Archivo Español de Arte, 335, Madrid, pp. 245-260.

Tormo, Elías (1909): “Al Sr. Serrano Fatigati sobre Escultura en Madrid y sobre deudos del Conde Duque (los Felípez de Guzmán)”. En: Boletín de la Sociedad Española de Excursiones, 17, 4, Madrid, pp. 291-312.

Tormo, Elías (1910): "Miscelánea de escultura del siglo XVII en Madrid”. En: Boletín de la Sociedad Española de Excursiones, 18, 3, Madrid, pp. 113-126.

Viceconte, Milena (2013): Il duca de Medina de las Torres (1600-1668) tra Napoli e Madrid: mecenatismo artistico e decadenza della monarchia (Tesis defendida en la Universitat de Barcelona en julio de 2013).

Fecha de recepción: 15-I-2017

Fecha de aceptación: 19-VI-2017 